\title{
Obesity, immunology and obesity surgery
}

\begin{abstract}
Abbreviations: T2DM, type2 diabetes mellitus; WAT, white adipose tissue; IL-6, interleukin-6; PAI-1, plasminogen activator inhibitor-1; TF, tissue factor; VEGF, vascular endothelial growth factor; CRP, c-reactive protein; MCP-1, monocyte chemoattractant protein-1; GIS, gastrointestinal system; PP, pancreatic polypeptide; GIP, glucose dependent insulinotropic polypeptide; OX, oxyntomodulin; GHSR, growth hormone secretagogue receptor; SG, sleeve gastrectomy
\end{abstract}

\section{Introduction}

Obesity is a major health problem with an increasing incidence in both developed and underdeveloped countries. It causes both psychological problems and also series systemic disease in the cardiovascular, metabolic and pulmonary system. In this context it has been reported that Type2 Diabetes Mellitus (T2DM), hypertension, congestive heart failure, thromboembolism, hyperlipidemia, sleep apnea, osteoarthritis and certain inflammatory diseases. Obesity related insulin resistance and chronic inflammation is the main factor in development of the metabolic syndrome. ${ }^{1}$

\section{Adipose tissue as an immun organ}

Obesity is defined as energy surplus in the white adipose tissue (WAT). In the pathophysiology of the process, adipocyte hypertrophy and pro inflammatory activation of the immune cells that are dormant in the adipose tissue (especially lymphocytes and macrophages) play an important role. These cells cause an increase in pro-inflammatory cytokines in the circulation. The chronic low grade inflammation in the adipose tissue is called as "metabolic inflammation" and seems to play an important role in the pathogenesis of insulin resistance and T2DM. In the adipose tissue of the lean individual the pro-inflammatory cells are suppressed by the regulatory cells; furthermore, activation is through the Th2 lymphocytes and macrophages are of M2 phenotype and the adipocytes are of normal size. In the adipose tissue of obese individuals, immune suppression is not present, pro-inflammatory cells aggregate and there is boost of pro-inflammatory cytokine production. Macrophage phenotype shifts to M1 and adipocytes are hypertrophic. ${ }^{2,3}$

Previously adipose tissue has been considered to be an organ for storage of triglycerides. However; currently it has been proven to be a metabolically active endocrine organ. In addition to all, it secretes many immunomodulatory factors that play major role in metabolic and vascular biology. It has previously been shown that Tumor necrosis factor $\alpha(\mathrm{TNF}-\alpha)$ and an appetite controlling hormone leptinwere to be secreted from the adipocytes. Recently, it has been shown that more than 50 hormones and signaling molecules are being secreted from the adipocytes and they are all together classified as "adipokines". Adipokines show endocrine, paracrine and autocrine effects that include many physiologic events such as energy and glucose metabolism and immune function. The adipokines; that play an important role in atherogenesis and development of insulin resistance; have many diversities in terms of properties. Leptin, adiponectin and resistin are proteins that have hormone-like properties; TNF- $\alpha$ and Interleukin-6 (IL-6) are classical cytokines; Plasminogen Activator Inhibitor-1(PAI-1) and Tissue Factor (TF) are vascular hemostasis
Volume 4 Issue 2 - 2016

\author{
Fusun Ozmen,' M Mahir Ozmen² \\ 'Department of Basic Oncology, Hacettepe University, Turkey \\ ${ }^{2}$ Department of Surgery, Hacettepe University, Turkey
}

Correspondence: M Mahir Ozmen, Professor of Surgery,

Department of Surgery, Medical School, Hacettepe University, 06100, Ankara-Turkey, Fax +90312310407I,

Email mahir.ozmen@hacettepe.edu.tr

Received: January 14, 2016 | Published: January 29, 2016

proteins, angiotensinogen is a blood pressure regulator; Vascular Endothelial Growth Factor (VEGF) is anangiogenic stimulant and C-Reactive protein (CRP) is an acute phase reactant.

\section{Adipokines and "Diabesity"}

$90 \%$ of the patients with T2DM have a BMI over $23 \mathrm{~kg} / \mathrm{m}^{2}$. The relative risk of developing T2DM in an obese individual is 10 times more than normal among the female and 11.2times in the male patients. The risk of diabetes considerably increases in early weight gain, abdominal obesity and in female with a history of gestational diabetes. The term diabesity defines the effects of excess fat on many organs involved in glucose homeostasis. Adipose dysfunction will eventually result in cardiovascular disease and mortality due to metabolic disease state caused by pathogenic mediators and lipotoxicity.Insulin resistance seems to play a major role in these conditions. ${ }^{4}$ Among the anti-inflammatory factors that are secreted from the adipose tissue are TGF- $\beta$, IL-10, IL-13, IL-1 receptor antagonist (IL-1ra) and apelin. On the other hand pro-inflammatory factors that are secreted are TNF- $\alpha$, IL-6, leptin, visfatin, resistin, angiotensin II, PAI-1, monocyte chemoattractant protein-1 (MCP-1).

The cytokines in the anti-inflammatory group show their own physiologic effects however, pro-inflammatory factors play a direct or indirect role in the insulin resistance of the organism. ${ }^{5,6} \mathrm{TNF}-\alpha$, IL6 , visfatin, resistin, PAI-1, MCP-1 expression is also observed in the adipose tissue related activated macrophages. TNF- $\alpha$, IL- 6 , resistin and other pro-inflammatory cytokines are responsible for the induction and generation of acute inflammatory response observed in obesity. Therefore; adipokines that include pro-inflammatory cytokines and chemokines activate certain intracellular pathways and result in insulin resistance, T2DM and metabolic complications observed in obese individuals. $^{7-9}$ The change in the adipose tissue mass of the obese individuals lead to changes in the circulating adipokine levels. Together with the weight loss or weight gain of the individuals due to certain reasons leads to changes in the adipokine levels and lead to changes in the metabolic and inflammatory parameters.

The treatment options for obesity such as diet, exercise and bariatric surgery lead to changes in the levels of these parameters to certain degrees. Surgical therapy of obesity is one option that gives the best result in terms of sustaind weight loss. ${ }^{10}$ Weight loss leads to changes in the immune system cells dormant in the adipose 
tissue. Following Roux NY Gastric Bypass in morbidly obese female patients; the macrophages present in the adipose tissue was observed to change from a pro-inflammatory phenotype to an anti-inflammatory phenotype. ${ }^{11}$

Resistin's name come from the its role in the pathogenesis of insulin resistance. In the rodents its secreted from the white adipose cells however; in humans its secreted from the adipose tissue as well as mononuclear immune cells such s macrophages. Resistin level in both rodents and human were found to be increased by obesity. The role of this adipokine in the pathogenesis of insulin resistance and glucose metabolism is controversial. Some researchers have postulated that the increased levels of resistin was correlated with obesity, increased insulin resistance and T2DM. However, some other researchers have not confirmed such a relationship. It has been suggested that resistin plays a more pronounced role during the inflammatory process in humans. In recent studies it has been shown that TNF- $\alpha$, IL- 6 and IL-1 $\beta$ induce the release of resistin from the mononuclear cells which causes and increased secretion of resistin in an autocrine fashion (positive feed-back). Resistin competes with lipopolysaccharide in order to bind the Toll-Like Receptor-4 and joinsthe imflammatory reaction through the $\mathrm{NF \kappa B}$ pathway and induce the secretion of TNF- $\alpha$, IL-6, IL-12,IL-1 $\beta$ and adhesion molecules (ICAM-1, VCAM1, E-selectin). ${ }^{12}$

TNF- $\alpha$ : It is one of the first cytokines that is found to be secreted from the adipocytes. It contribute to how grade inflammation in obese individuals together with other cytokines. It has been shown to contribute in pathogenesis of insulin resistance in animal models however it was not yet confirmed in humans.

IL-6: It is the first cytokine considered to be a biomarker in insulin resistance and cardiovascular disease. It is secreted from the adipose tissue and skeletal muscle. IL-6 serum levels are increased in obese individuals and its levels are reduced parallel to weight loss. However, the role of this adipokine in obesity and related disease is still unclear. ${ }^{13}$

\section{Obesity and GIS hormones}

Gastrointestinal system (GIS) is considered as the largest endocrine organ and the peptides that are secreted from GIS play role in gastrointestinal motility, secretion and absorption. Some of these hormones are also present in the central nervous system and enteric system as well. There are a variety of gastrointestinal peptides and they are either called hormone or neurocrine according to their function. Furthermore, these neurocrines have a paracrine action as well. Some of the gastrointestinal peptides that are considered to have hormone characteristics include Ghrelin, Cholecystokinin, Secretin, Peptide YY, Somatostatin, Glucagon Like Peptide-1 (GLP1), Pancreatic Polypeptide (PP), Glucose Dependent Insulinotropic Polypeptide (GIP) and oxyntomodulin (OX). Neuropeptide Y, Vasoactive Intestinal Peptide, Neurotensin are some of the peptides with neurocrine properties. These peptides enter the blood circulation following secretion from the gastrointestinal tract and act directly upon the brain through the circus ventricular organs (CVO). Information regarding the body energy storage is sent to the central nervous system by adipokines such as leptin, GIS hormones such as ghrelin. According to the signals received, hypothalamus secretes catabolic or anabolic mediators in order to constitute energy metabolism.

Ghrelinis one of the GIS peptides that act as a hormone. This 28 aminoasit long polypeptide is derived from peptide pre-proghrelin. It is secreted from gastric fundus and small bowel and it controls the appetite of the individual.It exerts its facts through the Growth Hormone Secretagogue receptor (GHSR); it binds to GSHR and penetrates the blood brain barrier. It affects the arcuate nucleus at the basal hypothalamus and surpasses the proopiomelanocortin and increases Neuropeptide YY. This constitutes the largest stimulus for increased appetite ${ }^{14}$ Great portion of the serum ghrelin is secreted by the ghrelin cells in the oxyntic glands of the fundic mucosa. These cells also express chrono-genes called PRR1 and PER2. The daylong expression follows an ultradian rhythm and usually peaks just before the meals which increases appetite. The increased ghrelin level decreases following the meal. ${ }^{15}$ chronic utilization of ghrelin led to hyperphagia and adipose tissue accumulation in experimental models.

Ghrelin also affects the glucose metabolism. It increases the secretion of insulin antagonist; glucagon and also the decreases the level of insulin sensitizing hormone; Leptin; therefore leads to an increase in the plasma glucose levels. Furthermore; it inhibits the hepatic insulin activity by blocking Phosphotidyl inositol-3 kinase and also inhibits the insulin secretion. ${ }^{16,17}$ Serum ghrelin levels in human are inversely correlated with the adiposity, body mass index (BMI) and changes in the body weight. Ghrelin level in the obese individuals is low however, weight reduction with diet causes an increase in ghrelin level. The effect of bariatric surgery on ghrelin is controversial. The ghrelin increase as expected with weight loss is not always observed following bariatric surgery. Furthermore there are studies showing a decrease in basal ghrelin levels. The reason for these variations are not known but may most probably be due to differences in the surgical technique..$^{18}$ Obesity and related disease are currently a worldwide pandemic. Conventional therapies such as diet, behavioral therapy, physical exercise and pharmacotherapy alone or in combination provide only a modest success rate and weight regain is observed after a period of time. Morbidly obese individuals especially respond poorly to these conventional therapies.

\section{The effects of bariatric surgical procedures}

The BMI over $30 \mathrm{~kg} / \mathrm{m}^{2}$ are considered as obese. Obesity is classified into three grades; BMI between 30-34.4 is called Grade I obesity, BMI between $35-39.9 \mathrm{~kg} / \mathrm{m}^{2}$ is considered to be Grade II obesity and BMI $\geq 40$ is considered to be Grade III obesity. Weight reduction surgery which is referred to as bariatric surgery is currently considered as an effective therapeutic modality for long-term weight control and also resolution of the co-morbid conditions such as T2DM. There are several bariatric surgical procedures for the treatment of morbid obesity. These procedures have been initially performed through laparotomy however in the minimal invasive era all procedures are currently being performed laparoscopically.

The basic mechanisms of the surgery include restrictive (Sleeve Gastrectomy [SG], Adjustable Gastric Banding [AGB]), malabsorptive (Dudenojejunal Bypass [DJB]) and combined techniques (Biliopancreatic Diversion[BPD], Bilopancreatic Diversion-Duodenal Switch [BPD-DS] or modifications (including SADI, SIPS etc), Roux en-Y Gastric Bypass [RYGB] and mini gastric Bypass $[\mathrm{MGB}])$. Each one of these procedures cause a certain change in the gastrointestinal anatomy and therefore cause different results. In sleeve gastrectomy fundus is resected and the stomach is tailored in to a narrow tube therefore a reduction in food intake is aimed. The resection of the fundus of the stomach is thought to be responsible for the reduction of the appetite. In RYGB, BPD, MGB, the chymus is transferred to distal small bowel bypassing the duodenum and proximal part of the small bowel. The expression of the GIS hormones 
from these region is increased. The caloric restriction and change in the GIS hormones following bariatric surgery is the responsible factor for the weight reduction and resolution of the metabolic disorders. There are two observations that support this hypothesis; first one is the fact that although there is negative energy balance and weight loss the appetite does not increase and in fact it decreases as well as the food intake also decreases. Second observation is the fact that T2DM removes almost instantly following bariatric surgery even before the initiation of the weight loss. ${ }^{1}$ Nevertheless; there are no clearcut indications for the selection of different procedures in different conditions and patient selection for specific procedures and research still continues to elucidates these demands.

SG is a vertical gastrectomy in which the fundus is resected totally. RYGB includes formation of a small gastric pouch followed by formation of a gastroeneterostomy $50 \mathrm{~cm}$ distal to the ligament of Trietz. Biliary limb is anatomized $100 \mathrm{~cm}$ distal to the gastroenterostomy and therefore $50 \mathrm{~cm}$ proximal gut is bypassed. ${ }^{1}$ In $\mathrm{MGB}$ (or one anastomosis gastric bypass, OAGB) gastric pouch is formed along the lesser curvature which is $50-75 \mathrm{ml}$ ( $12 \mathrm{~cm}$ or longer) and a Bilroth II gastroenetrostomy is performed in $150-350 \mathrm{~cm}$ distal to the ligament of Trietz. Therefore, it is clearly seen that RYGB and MGB are technically combined procedures leading to reduction in gastric volume and also anastomosis causing malabsorption. MGB is being increasingly popular among the surgeons because of shorter operative times and better weight reduction when compared to RYGB. ${ }^{19}$

In SG the gastric volume is limited to $60-150 \mathrm{~m}$ and gastric emptying becomes 2-3times more rapid. Therefore; duodenum and small bowel is exposed to chymus more rapidly. In RYGB the gastric volume is $15-30 \mathrm{ml}$ and the chymus bypasses $90 \%$ of the stomach and duodenum and proximal small bowel and it enters directly to the distal small bowel and the bile empties in to the jejunum. In MGB gastric volume is reduced $50-75 \mathrm{ml}$, chymus bypasses $80 \%$ of the gastric volume and it directly enters the proximal ileum (or distal jejunum) and bypasses almost the entire jejunum. Elemental nutrients such as glucose and amino acids are absorbed from the jejunum and therefore jejunum is an important part of bowel. Therefore the effect of these different surgical techniques on adipokines, cytokines, GIS hormones and T2DM are expected to be different from each other.

There is limited number of research in the field and the results are controversial. In certain studies the ghrelin level following SG is found to be reduced ${ }^{20}$ Harvey et al have analyzed the studies that have investigated the ghrelin level following SG and RYGB. Twenty three studies have analyzed and found controversial results; some of which reported an increase and the others reported a decrease. In some of these studies the postoperative follow-up periods were different and in the others the methods of detection were different. There is no consensus regarding the effects of bariatric surgery on serum ghrelin levels. 5 studies regarding the effects of sleeve gastrectomy on ghrelin have been analyzed and a reduction in serum ghrelin levels that were significantly higher than that following RYGB and AGB(adjustable gastric bant) was found in all of them. Insulin levels were found to be reduced following these surgical procedures. In T2DM patients; glycemic control had improved and insulin levels had decreased in early postoperative periods; even before observing a significant weight reduction following SG and RYGB. Leptin which is one of the adipokines is found to be decreased following RYGB however, its reduction was not significantly different from the reduction observed following diet/exercise programs. The changes in the adiponectine levels following bariatric surgery have shown variations. ${ }^{21,22}$

\section{Conclusion}

Obesity has many immunologic changes. Bariatric surgery results in certain level of changes on hormones also. Currently, there is only limited number of well-designed study evaluating the effects of bariatric surgery on hormonal and metabolic parameters of the patients mostly on roux $n \mathrm{Y}$ gastric bypass. Up to our knowledge there is no study investigating the effects of $\mathrm{MGB}(\mathrm{OAGB})$ or Duodenal Switch (SADI, SIPS etc) on adipokines, cytokines, GIS hormones and metabolic parameters of the patients. Therefore, research investigating the endocrine, humoral and metabolic effects of surgical procedures such as MGB which is a novel and technically less demanding bariatric procedure; is needed. In general, if a consensus can be reached regarding the effects of bariatric procedures, it will enable the physicians to reach certain guidelines to allocate patients into suitable bariatric procedures according to their accompanying diseases.

\section{Acknowledgements}

None.

\section{Conflict of interest}

The author declares no conflict of interest.

\section{References}

1. Finelli C, Padula MC, Martelli G, et al. Could the improvement of obesity-related co-morbidities depend on modified gut hormones secretion? World J Gastroenterol. 2014;20(44):16649-16664.

2. Ouchi N, Parker JL, Lugus JJ, et al. Adipokines in inflammation and metabolic disease. Nat Rev Immunol. 2011;11(2):85-97.

3. Han JM, Levings MK. Immune regulation in obesity-associated adipose inflammation. J Immunol. 2013;191(2):527-532.

4. Devanoorkar A, Kathariya R, Guttiganur N, et al. Resistin: a potential biomarker for periodontitis influenced diabetes mellitus and diabetes induced periodontitis. Dis Markers. 2014;2014:930206.

5. Makki K, Froguel P, Wolowczuk I. Adipose Tissue in Obesity Related Inflammation and Insulin Resistance: Cells, Cytokines and Chemokines. ISRN Inflamm. 2013;2013:139239.

6. Odegaard JI, Chawla A. Pleiotropic actions of insulin resistance and inflammation in metabolic homeostasis. Science. 2013;339(6116):172177.

7. Shoelson SE, Herrero L, Naaz A. Obesity, inflammation, and insulin resistance. Gastroenterology. 2007;132(6):2169-2180.

8. Lee H, Lee IS, Choue R. Obesity, Inflammation and Diet. Pediatr Gastroenterol Hepatol Nutr. 2013;16(3):143-152.

9. Blüher M, Klöting N, Wueest S, et al. Fas and FasL expression in human adipose tissue is related to obesity, insulin resistance, and type 2 diabetes. $J$ Clin Endocrinol Metab. 2014;99(1):E36-E44.

10. Santos J, Salgado P, Santos C, et al. Effect of Bariatric Surgery on Weight Loss, Inflammation, Iron Metabolism, and Lipid Profile. Scand J Surg. 2014;103(1):21-25.

11. Aron WJ, Tordjman J, Poitou C, et al. Human adipose tissue macrophages:m1 and $\mathrm{m} 2$ cell surface markers in subcutaneous and omental depots and after weight loss. J Clin Endocrinol Metab. 2009;94(11):4619-4623

12. Versini M, Jeandel PY, Rosenthal E, et al. Obesity in autoimmune diseases: not a passive bystander. Autoimmun Rev. 2014;13(9):981-1000.

13. Aguilar-Valles A, Inoue $\mathrm{W}$, Rummel $\mathrm{C}$, et al. Obesity, adipokines and neuroinflammation. Neuropharmacology. 2015;96(Pt A):124-134. 
14. Cheung CK, Wu JC. Role of Ghrelin in the Pathophysiology of Gastrointestinal Disease. Gut Liver. 2013;7(5):505-512.

15. Tewari N, Awad S, Lobo DN. Regulation of food intake after surgery and the gut brain axis. Curr Opin Clin Nutr Metab Care. 2013;16(5):569575 .

16. Karra E, Yousseif A, Batterham RL. Mechanisms facilitating weight loss and resolution of type 2 diabetes following bariatric surgery. Trends Endocrinol Metab. 2010;21(6):337-344.

17. Andrade S, Pinho F, Ribeiro AM, et al. Immunization against active ghrelin using virus-like particles for obesity treatment. Curr Pharm Des. 2013;19(36):6551-6558.

18. Sala PC, Torrinhas RS, Giannella-Neto D, et al. Relationship between gut hormones and glucose homeostasis after bariatric surgery. Diabetol Metab Syndr. 2014;6(1):87.
19. Mahawar KK, Jennings N, Brown J, et al. "Mini" gastric bypass: systematic review of a controversial procedure. Obes Surg. 2013;23(11):1890-1898.

20. Dimitriadis E, Daskalakis M, Kampa M, et al. Alterations in gut hormones after laparoscopic sleeve gastrectomy: a prospective clinical and laboratory investigational study. Ann Surg. 2013;257(4):647-654.

21. Harvey EJ, Arroyo K, Korner J, et al. Hormone changes affecting energy homeostasis after metabolic surgery. Mt Sinai J Med. 2010;77(5):446465 .

22. Chronaiou A, Tsoli M, Kehagias I, et al. Lower ghrelin levels and exaggerated postprandial peptide-YY, glucagon-like peptide-1, and insulin responses, after gastric fundus resection, in patients undergoing Roux-en-Y gastric bypass: a randomized clinical trial. Obes Surg. 2012;22(11):1761-1770. 